\title{
Strategies for the Identification and Prevention of Survey Fraud: Data Analysis of a Web-Based Survey
}

Mandi Pratt-Chapman ${ }^{1}, \mathrm{PhD} ;$ Jenna Moses $^{2}, \mathrm{BA} ;$ Hannah Arem ${ }^{3}, \mathrm{PhD}$

${ }^{1}$ GW Cancer Center, The George Washington University, Washington, DC, United States

${ }^{2}$ Milken Institute School of Public Health, The George Washington University, Washington, DC, United States

${ }^{3}$ Healthcare Delivery Research, Medstar Health Research Institute, Washington, DC, United States

Corresponding Author:

Mandi Pratt-Chapman, $\mathrm{PhD}$

GW Cancer Center

The George Washington University

2600 Virginia Ave

\#300

Washington, DC, 20037

United States

Phone: 12029945502

Email: mandi@email.gwu.edu

\section{Abstract}

Background: To assess the impact of COVID-19 on cancer survivors, we fielded a survey promoted via email and social media in winter 2020. Examination of the data showed suspicious patterns that warranted serious review.

Objective: The aim of this paper is to review the methods used to identify and prevent fraudulent survey responses.

Methods: As precautions, we included a Completely Automated Public Turing test to tell Computers and Humans Apart (CAPTCHA), a hidden question, and instructions for respondents to type a specific word. To identify likely fraudulent data, we defined a priori indicators that warranted elimination or suspicion. If a survey contained two or more suspicious indicators, the survey was eliminated. We examined differences between the retained and eliminated data sets.

Results: Of the total responses $(\mathrm{N}=1977)$, nearly three-fourths $(\mathrm{n}=1408)$ were dropped and one-fourth $(\mathrm{n}=569)$ were retained after data quality checking. Comparisons of the two data sets showed statistically significant differences across almost all demographic characteristics.

Conclusions: Numerous precautions beyond the inclusion of a CAPTCHA are needed when fielding web-based surveys, particularly if a financial incentive is offered.

(JMIR Cancer 2021;7(3):e30730) doi: 10.2196/30730

\section{KEYWORDS}

cancer survivors; pandemic; COVID-19; fraudulent responses; survey; research methods; cancer patients; fraud; CAPTCHA; data integrity; online surveys

\section{Introduction}

The COVID-19 pandemic resulted in significant delays to health care administration. To assess the impact of the pandemic on cancer survivors in the United States, the study team fielded a survey in the winter of 2020. The survey was promoted via email and, briefly, via social media. The volume of results in a short time period suggested that the data should be reviewed for fraudulent responses.
Social media can be an efficient way to disseminate web-based surveys [1-5]. According to the Pew Research Center, in 2021, $72 \%$ of adults in the United States were estimated to use at least one form of social media [6]. However, ensuring data integrity of studies when using social media remains a challenge. This study describes the data integrity methods used to identify fraudulent and suspicious data in a web-based survey that was briefly open to the public via social media. 


\section{Methods}

\section{Participant Sample}

We recruited cancer survivors primarily via an email request sent to physician liaisons and cancer registrars at institutions accredited by the Commission on Cancer (CoC). The study invitation, which came directly from the $\mathrm{CoC}$, asked recipients to forward the invitation to their cancer center survivorship coordinator, who in turn was asked to forward the invitation to patients. Emails were sent on October 13, 2020, followed by two reminders, each 1 week apart. In addition, the study team disseminated the survey to community partners on October 8 , 2020; posted on the Association of Community Cancer Centers eXchange and Association of Oncology Social Work listservs; and included the survey link in a George Washington University newsletter to health care professionals.

\section{Incentives}

Participants were asked to complete a 20-minute survey and were told they would receive a US \$25 gift card to thank them for their time.

\section{Precautions}

To dissuade bots, we included a Completely Automated Public Turing test to tell Computers and Humans Apart (CAPTCHA), a question asking how the participant heard about the survey, time stamps, open-ended questions, and pairs of items that could be compared for consistency. After receiving over 1000 responses in the first 3 days after opening the survey, we examined the data and identified suspicious patterns. We then removed all links from social media and added additional precautions based on extant literature about optimizing valid responses for public-access surveys [7-9]: including a hidden item that could only be detected by bots, requiring participants to retype a word, and requiring participants to confirm their understanding that fraudulent responses would not be compensated.

\section{Measures}

Our survey questions included demographics and health history: age, sex, and gender identity; sexual orientation; race/ethnicity; marital status; household size; education; income; age at diagnosis; cancer stage; cancer type; employment status; and insurance type. We also included questions related to COVID-19 and patient-reported outcomes.

\section{Data Cleaning}

Data were exported from Research Electronic Data Capture (REDCap) and analyzed in SAS 9.4 (SAS Institute). As of Thursday, December 3, 2020, we had received 1977 responses. We thus developed criteria to identify suspicious and fraudulent data.

We began by eliminating those who were ineligible: respondents who were living outside of the United States, had stage 0 cancer, had no cancer diagnosis $(n=83)$, or reported that they had only nonmelanoma skin cancer $(n=46)$ [10]. We then eliminated respondents who were missing data on $\geq 35 \%$ of survey questions $(n=149)$. Next, we excluded respondents who reported contradictory responses, including discordant gender (eg, both cisgender male and cisgender female status) $(n=12)$ and discordant sex assigned at birth with anatomical site of cancer (eg, cisgender male with uterine cancer) $(n=37)$.

We analyzed irregularities in the remaining data $(n=1650)$ and eliminated responses that contained two or more suspicious indicators (Table 1). Criteria for a suspicious indicator included differences between reported and calculated age or reported and calculated time between treatment and diagnosis; report of a type of cancer that is very rare for the respondent's age group; incongruent patterns of hearing about the survey relative to distribution dates; suspicious open-text responses (including fake addresses); repeat email addresses; and unusual time stamps. Table 1 presents a summary of the types of fraudulent and suspicious responses, and Figure 1 shows the elimination sequence.

We sent emails to all respondents excluded from the final data set to alert them that their responses had not passed a quality check, and we welcomed them to reach out to the study team with any questions. We received only 1 response, which said: "Why." We also emailed all of the respondents who were retained in the data set and instructed them on how to claim their incentive. We received 1 response from a person who did not recall participating in the study. As additional quality control, we reviewed a subset of data for respondents who indicated hearing about the survey from a specific community partner. Of the 35 respondents who indicated hearing about the survey from this partner, we excluded 30. Upon member checking, all 5 participants retained in the data set were confirmed as clients of the community partner, and only 1 of the excluded respondents was a legitimate client. 
Table 1. Types of fraudulent or suspicious data identified in eliminated survey responses $(n=1081){ }^{a}$

\begin{tabular}{|c|c|}
\hline Description & Value, $\mathrm{n}(\%)$ \\
\hline $\begin{array}{l}\text { Year of birth is reported as 2020, or reported age and age calculated from reported date of birth are different by more than } 1 \\
\text { year }\end{array}$ & $250(17.8)$ \\
\hline Reported age is $<40$ years and cancer type is rare for those aged $<40$ years & $283(20.1)$ \\
\hline Respondents indicate a survey source prior to dissemination of the survey from that source & $820(58.2)$ \\
\hline Open-ended comments focus on information technology rather than answering the question asked & $56(4)$ \\
\hline Open-ended telehealth comments are duplicates & $34(2.4)$ \\
\hline Final open-ended suggestion responses are duplicates & $107(7.6)$ \\
\hline Email addresses are duplicates & $20(1.4)$ \\
\hline Time since diagnosis is $<2$ years, but time since treatment is $2-5$ years & $11(0.8)$ \\
\hline Time since diagnosis is $\leq 5$ years, but time since treatment is $>5$ years & $57(4)$ \\
\hline $\begin{array}{l}\text { Suspicious survey time (at least } 10 \text { surveys completed in succession within } 5 \text { minutes of each other or completed between } \\
\text { midnight and } 4 \text { AM EST) }\end{array}$ & $986(70)$ \\
\hline $\begin{array}{l}\text { Email/address is suspicious (for email: at least } 10 \text { random numbers or letters in a row, or strange punctuation or capitalization; } \\
\text { for address: incomplete address, address of a business, address is not real, address includes quotation marks, or pattern of } \\
\text { strange capitalization or spacing) }\end{array}$ & $166(11.8)$ \\
\hline $\begin{array}{l}\text { Name/suffix is suspicious (first and last name flipped, part of last name in first name field or vice versa, male suffix and female } \\
\text { name, random letters or numbers in suffix field) }\end{array}$ & $78(5.5)$ \\
\hline
\end{tabular}

${ }^{\mathrm{a}}$ Individuals could be counted in as many indicators as their responses suggested; thus, the $\mathrm{n}$ values do not add up to the total of excluded data.

Figure 1. Flow chart of survey response elimination. REDCap: Research Electronic Data Capture.

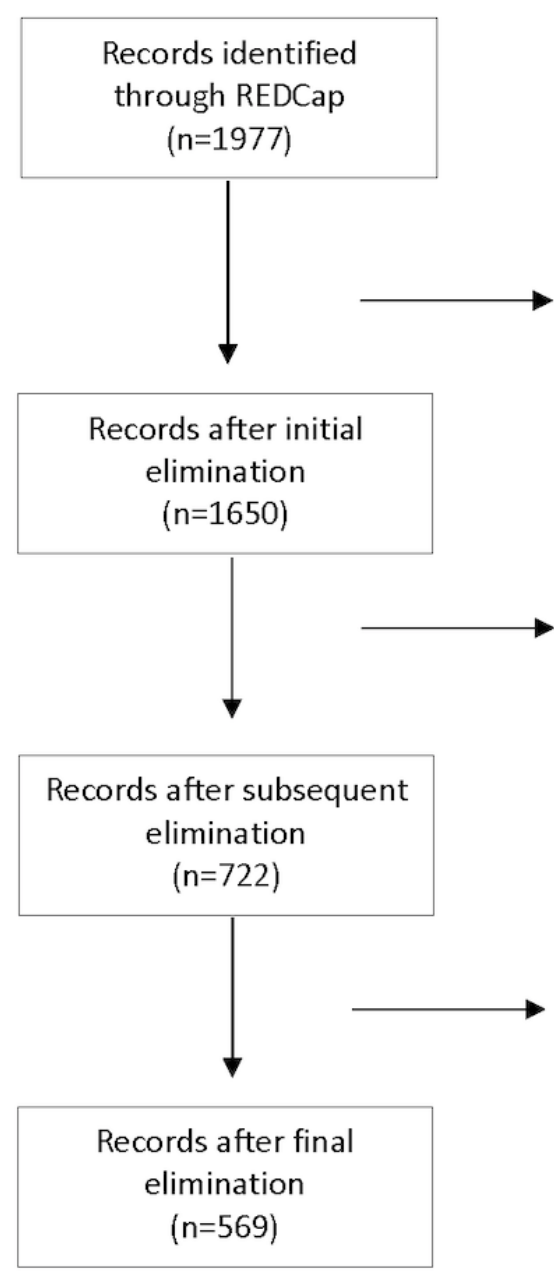

Initial exclusions based on noneligibility ( $\mathrm{n}=327)$

- Noneligibility based on study protocol (eg, lived outside US, reported only non-melanoma skin cancer) $(n=129)$

- Only reported demographic data $(n=149)$

- Reported incompatible genders (e.g. reported both cisgender male and transgender male) $(n=12)$

- Gender not congruent with cancer type (e.g. cisgender male with ovarian cancer) $(n=37)$

Exclusions based on quantitative criteria ( $n=928$ )

- Had two or more suspicious indicators $(n=928)$

Exclusions based on qualitative criteria $(n=153)$

- Two or more suspicious indicators after identifying suspicious names, emails, and addresses ( $n=145)$

- Name and gender not congruent plus one other suspicious indicator $(n=8)$ 


\section{Data Analysis}

After eliminating responses deemed as fraudulent, we used means and frequencies to create a demographics table comparing respondents who were included with those who were excluded. We used chi-square or Fisher exact tests to examine differences between groups.

\section{Ethical Review}

This study was deemed exempt by the George Washington University Institutional Review Board (IRB) (NCR202819).

\section{Results}

Of the total sample ( $\mathrm{N}=1977), 1408$ responses were excluded (327 due to ineligibility and 1081 due to suspicious responses) and 569 were retained. Most surveys eliminated were dated October 9-11, $2020(n=1072)$. These dates align with the period when the survey link was posted on social media.
Comparisons of retained and excluded respondents showed statistically significant differences across most demographic characteristics (Table 2). There were lower rates of cisgender male, transgender/gender fluid/two-spirit identification $(P<.001)$ and higher rates of cisgender female identification $(P<.001)$ among retained versus excluded respondents. There was a higher prevalence of straight-identifying respondents in the retained sample versus the excluded sample $(P<.001)$. There were lower rates of respondents reporting Native American/Alaska Native/Pacific Islander race/ethnicity $(P<.001)$ and higher rates of those reporting White race/ethnicity in the retained sample versus the excluded sample $(P<.001)$. The numbers of single individuals were similar in the two samples, but higher rates of divorced/separated and widowed people were observed in the retained sample versus the excluded sample $(P<.001)$. There were higher rates of college completion and graduate school as well as annual incomes greater than US $\$ 100,000$ among the retained sample versus the excluded sample $(P<.001)$. The mean age of the retained sample was significantly older (56 vs 42 years old, $P<.001)$. 
Table 2. Differences between respondents in the retained and excluded samples.

\begin{tabular}{|c|c|c|c|}
\hline Characteristic & Retained sample $(\mathrm{n}=569)$ & Excluded sample $(\mathrm{n}=1081)$ & $P$ value \\
\hline Current age (years), mean (SD) & $55.9(13.1)$ & $41.4(8.2)$ & $<.001$ \\
\hline \multicolumn{4}{|l|}{ Gender, $\mathbf{n}(\%)^{\mathbf{a}, \mathbf{b}}$} \\
\hline Cisgender male & $132(23.2)$ & $575(53.2)$ & $<.001$ \\
\hline Transgender male, transgender female, gender fluid, or two-spirit & $1(0.2)$ & $32(3.0)$ & $<.001$ \\
\hline Cisgender female & $399(70.1)$ & $463(42.8)$ & $<.001$ \\
\hline Other/prefer not to answer/Do not understand the question & $40(7.0)$ & $14(1.3)$ & $<.001$ \\
\hline Sexual orientation, $\mathbf{n}(\%)^{b}$ & & & $<.001$ \\
\hline Straight & $532(93.5)$ & $984(91.0)$ & \\
\hline Lesbian, gay, homosexual, bisexual/pansexual, queer, two-spirit & $23(4.0)$ & $89(8.2)$ & \\
\hline Other/prefer not to answer/do not understand the question & $14(2.5)$ & $8(0.7)$ & \\
\hline \multicolumn{4}{|l|}{ Race/ethnicity, n (\%) $)^{\mathbf{a}, \mathbf{b}}$} \\
\hline Asian & $19(3.3)$ & $58(5.4)$ & .06 \\
\hline Black & $83(14.6)$ & $200(18.5)$ & .045 \\
\hline Hispanic/Latinx & $42(7.4)$ & $90(8.3)$ & .50 \\
\hline Native American/Alaska Native/Pacific Islander & $17(3.0)$ & $83(7.7)$ & $<.001$ \\
\hline White & $411(72.2)$ & $677(62.6)$ & $<.001$ \\
\hline Partnership status, $\mathbf{n}(\%)^{b}$ & & & $<.001$ \\
\hline Single & $93(16.3)$ & $152(14.1)$ & \\
\hline Married/partnered & $388(68.2)$ & $884(81.8)$ & \\
\hline Divorced/separated & $60(10.5)$ & $37(3.4)$ & \\
\hline Widowed & $28(4.9)$ & $8(0.7)$ & \\
\hline Number of individuals in household, mean (SD) & $2.6(1.3)$ & $3.3(0.9)$ & $<.001$ \\
\hline Education, $\mathbf{n}(\%)^{\mathrm{b}}$ & & & $<.001$ \\
\hline Some high school or less & $17(3.0)$ & $38(3.5)$ & \\
\hline High school diploma or GED $/$ vocational school & $88(15.8)$ & $294(27.2)$ & \\
\hline Some college & $164(28.8)$ & $415(38.4)$ & \\
\hline Completed 4-year degree & $156(27.4)$ & $261(24.1)$ & \\
\hline Graduate school & $144(25.3)$ & $73(6.8)$ & \\
\hline Annual household income (US \$), $n(\%)^{b}$ & & & $<.001$ \\
\hline$<25,000$ & $59(10.4)$ & $46(4.3)$ & \\
\hline $25,001-50,000$ & $106(18.6)$ & $383(35.4)$ & \\
\hline $50,001-75,000$ & $124(21.7)$ & $375(34.7)$ & \\
\hline $75,001-100,000$ & $61(10.7)$ & $182(16.8)$ & \\
\hline$>100,000$ & $129(22.7)$ & $93(8.6)$ & \\
\hline I prefer not to answer & $90(15.8)$ & $1(0.09)$ & \\
\hline Age at cancer diagnosis (years), mean (SD) & $51.4(13.4)$ & $36.8(8.6)$ & $<.001$ \\
\hline Cancer stage, $n(\%)^{b}$ & & & $<.001$ \\
\hline I & $172(30.2)$ & $456(42.2)$ & \\
\hline II & $167(29.4)$ & $367(34.0)$ & \\
\hline III & $88(15.5)$ & 177 (16.4) & \\
\hline
\end{tabular}




\begin{tabular}{|c|c|c|c|}
\hline Characteristic & Retained sample $(\mathrm{n}=569)$ & Excluded sample $(\mathrm{n}=1081)$ & $P$ value \\
\hline IV & $62(10.9)$ & $51(4.7)$ & \\
\hline Unknown & $66(11.6)$ & $24(2.2)$ & \\
\hline \multicolumn{4}{|l|}{ Cancer type, $\mathbf{n}(\%)^{\mathrm{a}, \mathrm{b}}$} \\
\hline Melanoma & $26(4.6)$ & $57(5.3)$ & .53 \\
\hline Lung & $23(4)$ & $199(18.4)$ & $<.001$ \\
\hline Prostate & $37(6.5)$ & $90(8.3)$ & .19 \\
\hline Breast & $328(57.6)$ & $161(14.9)$ & $<.001$ \\
\hline Colorectal & $39(6.9)$ & $117(10.8)$ & .008 \\
\hline Kidney & $8(1.4)$ & $63(5.8)$ & $<.001$ \\
\hline Bladder & $8(1.4)$ & $83(7.7)$ & $<.001$ \\
\hline Blood cancer (leukemia, lymphoma, myeloma) & $44(7.7)$ & $82(7.6)$ & .92 \\
\hline Uterine/cervical & $32(5.6)$ & $160(14.8)$ & $<.001$ \\
\hline Thyroid & $31(5.5)$ & $91(8.4)$ & .03 \\
\hline Other & $62(10.9)$ & $13(1.2)$ & $<.001$ \\
\hline Time since cancer treatment (years), $n(\%)^{b}$ & & & $<.001$ \\
\hline$<2$ & $238(43.4)$ & $476(44.1)$ & \\
\hline $2-5$ & $168(30.7)$ & $488(45.2)$ & \\
\hline$>5$ & $142(25.9)$ & $116(10.7)$ & \\
\hline \multicolumn{4}{|l|}{ Cancer care status, $\mathbf{n}(\%)^{\mathrm{a}, \mathrm{b}}$} \\
\hline My cancer is in remission or no evidence of disease & $447(78.6)$ & $612(56.6)$ & $<.001$ \\
\hline I have chronic cancer & $77(13.5)$ & $240(22.2)$ & $<.001$ \\
\hline I am receiving palliative care & $30(5.3)$ & $253(23.4)$ & $<.001$ \\
\hline I am in hospice care & $0(0)$ & $60(5.6)$ & $<.001$ \\
\hline None of these apply to me & $42(7.4)$ & $39(3.6)$ & $<.001$ \\
\hline Part of a tribe or territory, $\mathrm{n}(\%)^{\mathrm{b}}$ & $41(7.2)$ & $397(38.1)$ & $<.001$ \\
\hline \multicolumn{4}{|l|}{ Employment status, $\mathbf{n}(\%)^{\mathrm{a}, \mathrm{b}}$} \\
\hline Retired & $198(34.8)$ & $48(4.4)$ & $<.001$ \\
\hline Paid work (full- or part-time) & $251(44.1)$ & $667(61.7)$ & $<.001$ \\
\hline Unpaid work (homemaker, volunteer) & $44(7.7)$ & $127(11.8)$ & .01 \\
\hline Unemployed & $77(13.5)$ & $247(22.9)$ & $<.001$ \\
\hline \multicolumn{4}{|l|}{ Insurance type, $\mathbf{n}(\%)^{\mathrm{a}, \mathrm{b}}$} \\
\hline Private insurance & $320(56.2)$ & $436(40.3)$ & $<.001$ \\
\hline Medicaid & $83(14.6)$ & $491(45.4)$ & $<.001$ \\
\hline Medicare & $210(36.9)$ & $633(58.6)$ & $<.001$ \\
\hline Tricare/COBRA $\%$ other & $48(8.4)$ & $64(5.9)$ & .054 \\
\hline I do not have health insurance & $31(5.5)$ & $45(4.2)$ & .24 \\
\hline Self-reported health, $\mathbf{n}(\%)^{b}$ & & & $<.001$ \\
\hline Excellent/very good & $165(29.0)$ & $375(34.7)$ & \\
\hline Good & $226(39.7)$ & $318(29.4)$ & \\
\hline Fair & $101(17.8)$ & $254(23.5)$ & \\
\hline
\end{tabular}




\begin{tabular}{cll}
\hline Characteristic & Retained sample $(\mathrm{n}=569)$ & $\frac{\text { Excluded sample }(\mathrm{n}=1081) \quad P \text { value }}{133(12.3)}$ \\
\hline Poor & $17(3.0)$ & 133.0 \\
\hline
\end{tabular}

${ }^{\mathrm{a}}$ Respondents could select multiple responses for this question.

${ }^{b}$ Responses may not add up to $n=569$ or $n=1081$ due to missing data or multiple responses.

${ }^{\mathrm{c}}$ GED: General Educational Development.

${ }^{\mathrm{d} C O B R A}$ : Consolidated Omnibus Budget Reconciliation Act.

The samples also differed in cancer stage, type, health status, and insurance coverage status. The retained sample reported more stage IV cancer and a higher percentage of breast cancer than the excluded sample. The excluded sample reported more lung, kidney, bladder, and uterine/cervical cancers than the retained sample $(P<.001)$. A greater percentage of the retained versus excluded sample reported completing treatment more than 5 years ago $(142 / 569,25.9 \%$, vs $116 / 1081,10.7 \% ; P<.001)$. A greater percentage of those in the retained sample indicated their cancer was in remission or had no evidence of disease $(447 / 569,78.6 \%$, vs $612 / 1081,56.6 \% ; P<.001)$, while a greater percentage of the excluded sample reported receiving palliative care $(253 / 1081,23.4 \%$, vs $30 / 569,5.3 \% ; P<.001)$ and hospice $(60 / 1081,5.6 \%$, vs $0 / 569,0 \% ; P<.001)$. A greater percentage of the retained sample reported having private insurance (320/569, 56.2\%), while more of the excluded sample reported having Medicaid (491/1081, 45.4\%) and/or Medicare (633/1081, $58.6 \%$ ). Finally, respondents in the retained sample were more likely to report their health as "good" $(226 / 569,39.7 \%$, vs $318 / 1081,29.4 \%$ ) and less likely to report their health as "poor" $(17 / 569,3.0 \%$, vs $133 / 1081,12.3 \%)$ compared to the excluded sample $(P<.001)$.

\section{Discussion}

\section{Principal Findings}

Numerous indications support the greater integrity of the data in the retained sample $(n=569)$ compared to the excluded sample $(n=1081)$. First, discordant data reported by the same respondent, such as the anatomical site of their cancer not being physically possible for their reported sex/gender, were clear signs of random survey completion. Second, the younger mean age of the excluded sample combined with cancers more likely to be diagnosed at a later age (eg, lung, kidney, and bladder cancers), more serious disease (chronic, receiving palliative care, or hospice), and poorer health is highly suspicious. Conversely, the higher self-reported diagnosis of breast cancer in the retained sample aligns with the authors' prior research experience in more easily recruiting breast cancer survivors than those with a history of other cancers.

This study contributes to the literature by providing guidance for identifying potentially fraudulent data. Importantly, use of screening questions and CAPTCHA was insufficient to dissuade fraudulent respondents. Consistent with past research, we found that examining repeated personal data across responses [11], duplicate open text responses [12], response inconsistency [12], and low-probability responses [12] helped to identify potentially fraudulent responses. Additionally, we found that examining differences between the retained and excluded samples bolstered our confidence in the retained sample (ie, demographic characteristics such as mean age and cancer type corresponded more closely with the demographics of participants in prior cancer survivorship research conducted by the authors as well as cancer statistics).

\section{Ethical Considerations}

Social media is an efficient and cost-effective method for health research. However, the potential for loss of data integrity must be weighed with the efficiency and cost-effectiveness [1-5]. The distance created between researchers and participants in internet survey-based research may lead to participants feeling less self-conscious about unethical behavior and more motivated to obtain incentives for which they are ineligible. Precautions to improve confidence in data integrity, however, may inadvertently prevent participation by eligible persons as well. For example, persons using the same computer who are eligible to participate in a study may be omitted from data based on their identical IP addresses. People with less technological savvy or visual challenges may be dissuaded from survey completion by the CAPTCHA. People whose first language does not match the language of the survey may be dissuaded due to instructions to type words in a language in which they are not facile. Finally, the capture of geographic location (IP address) in combination with multiple identifying questions has implications for the anonymity of respondents. Prevention and detection of fraudulent responses may, therefore, require increased justification for IRB review to collect geolocation and identifying data that would not otherwise be needed.

\section{Recommendations to Prevent Fraudulent Data}

To minimize bot contamination and reduce duplicate entries, precautions similar to those taken in this study are warranted. Additional recommendations include using software with fraud prevention and detection capabilities (eg, Qualtrics), capturing IP addresses, capturing time stamps for both start and stop times, including a required open text question, and distributing surveys only to closed groups on social media or avoiding social media altogether. If social media is used, financial incentives should be avoided. If providing financial incentives, (1) require participants to check a box indicating they acknowledge that responses from ineligible respondents or those who respond multiple times will not receive the financial incentive and downplay the incentive, and (2) indicate that investigators reserve the right to confirm eligibility by telephone (or other means) and include a required telephone number field.

\section{Recommendations to Identify Fraudulent Data}

Once data are collected, data integrity checks such as those in Table 2 can help researchers detect potentially fraudulent responses. In addition, the use of different trackable URLs for 
different dissemination channels may facilitate the identification of the dissemination source of suspicious data.

\section{Limitations}

The criteria used to eliminate responses were subjective, and it is impossible to know if all fraudulent data were removed. The authors erred on the side of potentially eliminating valid responses rather than retaining responses that were likely to be invalid. Limitations in our ability to detect potentially fraudulent responses included the inability to capture IP addresses or completion times.

\section{Conclusion}

Providing a survey incentive in combination with social media recruitment may increase the likelihood of fraudulent activity. CAPTCHA alone is unlikely to prevent fraudulent responses in internet-based research promoted on social media. Precautions to prevent and detect fraud are important for the validity of research findings. Ethical considerations of participant privacy and incentive payments should be weighed with data integrity concerns to ensure valid, meaningful health research results.

\section{Acknowledgments}

Funding for the study was provided by a Patient Centered Outcomes Research Institute Engagement Award (EADI-12744). Thank you to the Community Advisory Board for this project, including Katie Bathje, Benoit Blondeau, Cindy Cisneros, Ysabel Duron, Maureen Killackey, Larissa Nekhlyudov, Beth Sieloff, and Megan Slocum. Thank you to Ysabel Duron and Larissa Nekhlyudov for review and feedback on an earlier draft of this manuscript.

\section{Conflicts of Interest}

None declared.

\section{References}

1. Guillory J, Kim A, Murphy J, Bradfield B, Nonnemaker J, Hsieh Y. Comparing Twitter and online panels for survey recruitment of e-cigarette users and smokers. J Med Internet Res 2016 Nov 15;18(11):e288 [FREE Full text] [doi: 10.2196/jmir.6326] [Medline: 27847353]

2. Rosa C, Campbell ANC, Miele GM, Brunner M, Winstanley EL. Using e-technologies in clinical trials. Contemp Clin Trials 2015 Nov;45(Pt A):41-54. [doi: 10.1016/j.cct.2015.07.007] [Medline: 26176884]

3. Wozney L, Turner K, Rose-Davis B, McGrath PJ. Facebook ads to the rescue? Recruiting a hard to reach population into an Internet-based behavioral health intervention trial. Internet Interv 2019 Sep;17:100246 [FREE Full text] [doi:

10.1016/j.invent.2019.100246] [Medline: 31080751]

4. Topolovec-Vranic J, Natarajan K. The use of social media in recruitment for medical research studies: a scoping review. J Med Internet Res 2016 Nov 07;18(11):e286. [doi: 10.2196/jmir.5698]

5. Whitaker C, Stevelink S, Fear N. The use of Facebook in recruiting participants for health research purposes: a systematic review. J Med Internet Res 2017 Aug 28;19(8):e290. [doi: 10.2196/jmir.7071]

6. Social media fact sheet. Pew Research Center. URL: https://www.pewresearch.org/internet/fact-sheet/social-media/ [accessed 2021-05-21]

7. Pozzar R, Hammer MJ, Underhill-Blazey M, Wright AA, Tulsky JA, Hong F, et al. Threats of bots and other bad actors to data quality following research participant recruitment through social media: cross-sectional questionnaire. J Med Internet Res 2020 Oct 7;22(10):e23021. [doi: 10.2196/23021]

8. Ballard AM, Cardwell T, Young AM. Fraud detection protocol for web-based research among men who have sex with men: development and descriptive evaluation. JMIR Public Health Surveill 2019 Feb 04;5(1):e12344. [doi: 10.2196/12344]

9. Teitcher JEF, Bockting WO, Bauermeister JA, Hoefer CJ, Miner MH, Klitzman RL. Detecting, preventing, and responding to "fraudsters" in internet research: ethics and tradeoffs. J Law Med Ethics 2015 Apr;43(1):116-133 [FREE Full text] [doi: 10.1111/jlme.12200] [Medline: 25846043]

10. Adamo M, Dickie L, Ruhl J. SEER program coding and staging manual. Bethesda, MD: Department of Health and Human Services National Instiutes of Health; 2018.

11. Conrique BG, McDade-Montez E, Anderson PM. Detection and prevention of data fraud in a study of community college career technical education students. Community Coll J Res Pract 2019 Jun 13;44(9):690-693. [doi: $10.1080 / 10668926.2019 .1629126]$

12. Dewitt J, Capistrant B, Kohli N. Addressing participant validity in a small internet health survey (the Restore Study): Protocol and recommendations for survey response validation. J Med Internet Res 2018;20(4):1-1. [doi: 10.2196/preprints.7655]

\section{Abbreviations}

CAPTCHA: Completely Automated Public Turing test to tell Computers and Humans Apart

COC: Commission on Cancer

IRB: Institutional Review Board 
REDCap: Research Electronic Data Capture

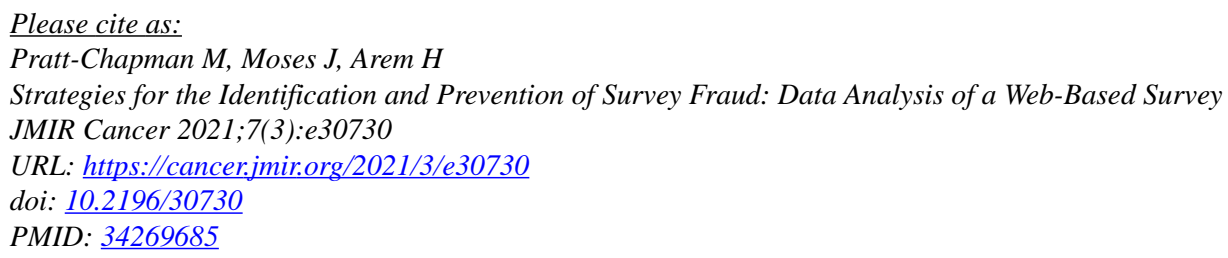

CMandi Pratt-Chapman, Jenna Moses, Hannah Arem. Originally published in JMIR Cancer (https://cancer.jmir.org), 16.07.2021. This is an open-access article distributed under the terms of the Creative Commons Attribution License (https://creativecommons.org/licenses/by/4.0/), which permits unrestricted use, distribution, and reproduction in any medium, provided the original work, first published in JMIR Cancer, is properly cited. The complete bibliographic information, a link to the original publication on https://cancer.jmir.org/, as well as this copyright and license information must be included. 